\title{
Joint Array Combining and MLSE for Single-User Receivers in Multipath Gaussian Multiuser Channels
}

\author{
Miguel A. Lagunas, Fellow, IEEE, Josep Vidal, Member, IEEE, and Ana I. Pérez-Neira, Member, IEEE
}

\begin{abstract}
The well-known structure of an array combiner along with a maximum likelihood sequence estimator (MLSE) receiver is the basis for the derivation of a space-time processor presenting good properties in terms of co-channel and intersymbol interference rejection. The use of spatial diversity at the receiver front-end together with a scalar MLSE implies a joint design of the spatial combiner and the impulse response for the sequence detector. This is faced using the MMSE criterion under the constraint that the desired user signal power is not cancelled, yielding an impulse response for the sequence detector that is matched to the channel and combiner response. The procedure maximizes the signal-to-noise ratio at the input of the detector and exhibits excellent performance in realistic multipath channels.
\end{abstract}

Index Terms-Adaptive antennas, multipath channels, multiuser/single-user receivers, wireless communications.

\section{INTRODUCTION}

A N ISSUE of paramount importance in mobile communications is the conflict between spectrum availability and the exponentially increasing demands for wireless services. This has led manufacturers to the search for the potentials associated with spatial diversity techniques so as to alleviate congestion problems. It has been shown lately [19] that the use of antenna arrays in a spatial division multiple access (SDMA) strategy leads to an important increase in system capacity and an important increase in system immunity to power variations. Practical results obtained through intensive simulations [22] have demonstrated that capacity increases in $200 \%$ when antenna arrays are utilized to reject interferences in the so-called spatial filtering interference rejection (SFIR) configuration.

On the other hand, technologic and economic requirements impose constraints on the complexity of the algorithms used in base stations. In this respect, optimal multichannel/multiuser receivers (which jointly detect all the $R$ users' signals present in the scenario) are often unaffordable structures, with computational complexity on the order of $O\left(M^{R}\right)$ for $M$-ary signal constellations. Therefore, some suboptimal solutions have appeared which deal with interference as if it were noise (single user receivers). Some effort has been made to unify spatial and temporal processing, and include the proper covariance matrix in the MLSE metrics [24]-[26], at the expenses of added com-

Manuscript received February 12, 1999; revised September 23, 1999. This work was supported in part by the European Commission under ACTS, AC347 SUNBEAM; the Spanish National Plan of Research, under Projects CICYT, TIC98-0412, and TIC98-0703; and by the AECI and Generalitat de Catalunya, under Project CIRIT, 1998SGR-00081.

The authors are with the Department of Signal Theory and Communications, Universitat Politècnica de Catalunya, 08034 Barcelona, Spain (e-mail: pepe@gps.tsc.upc.es).

Publisher Item Identifier S 0733-8716(00)09199-X. plexity. Performances are below the optimal (but good enough for some applications) and the complexity is on the order $O(R)$. In some cases [17], [21], the space and time processors are completely separated and estimated, and it is crucial that both are designed jointly and each one is assigned the appropriate role.

For the temporal processor, the maximum likelihood sequence estimator (MLSE), when designed for white noise and no interference, exhibits moderate complexity but it is quite sensitive to the presence of co-channel interferers or temporally correlated noise [1]. At the same time, the length of the estimated channel has a major impact in MLSE in terms of complexity and decoder delay. In summary, any preprocessing aiming at the reduction of the channel length and removal of co-channel interferers is welcome. One of the solutions which is still in use in the single receiver case [2] is to set a forward equalizer (FE) whose major objective is to reduce the channel length. The important drawback is the introduction of temporal correlation in the noise. An FE could also partially remove co-channel interferers if fractional sampling is used [17]. In practice, signals not having enough excess bandwidth cannot be used in such configuration and interferences are not cancelled. Both effects have a negative impact in the performance of the MLSE [3].

On the other hand, a spatial processor (array combiner) could reject late arrivals that introduce ISI as well as cancelling co-channel interferers, at the expense of a large number of sensors. It should be considered, however, that ISI is not a bad phenomenon when dealing with fading channels. If the amplitudes of the arriving rays are uncorrelated, delay spread provides time diversity that can be used properly with a time processor. One of the important features of the array combiner is that it does not introduce temporal correlation in the noise.

Therefore, if both time and space processors are used and jointly optimized, the resulting receiver is able to spatially filter the co-channel interferences and leave the task of ISI compensation to the time processor. Moreover, the spatial processor may cancel late signal arrivals, thus reducing the impulse response length seen by the MLSE block. One example of this joint approach is [21], where a space-time equalizer is split in two parts: an FE or a decision feedback equalizer follows the array combiner. In [16], a multisensor space-time equalizer removes co-channel interference and part of the ISI, while a vector MLSE block removes the residual ISI and detects symbols. However, since the noise at the input of the vector MLSE is not white the optimum metrics of the Viterbi algorithm are difficult to compute, so the performance of this approach is necessarily limited. Moreover, the estimation of the spatial and temporal processors is not done jointly. 
The approach proposed here takes advantage of the strength of both spatial combining and MLSE: a wide-band (or narrowband) array combiner plus sampler cancels the co-channel interference and yields white noise, while the MLSE block optimally deals with intersymbol interference and detects symbols. The parameters of both processors are estimated jointly using the MMSE criterion. As expected, the resulting impulse response seen by the MLSE block is the spatial filtering of the space-time propagation channel of the desired user, so the method will be called (in the sequel) matched desired impulse response, or MDIR. Of course, the ability to spatially filter out interferences depends on whether the number of undesired users is commensurate with the number of sensors.

This space-time receiver was first reported in [5]. The design used different constraints but the solutions finally obtained turned out to be similar to those published in [4] and presented here. Also, the basic concepts involved in these works can be found in [6], [3], and [2], where these concepts were applied in a different framework. The computation of the receiver is done generally for both fractionally spaced and symbol-time spaced samplers. Complexity reduction is a primary issue in wideband receivers [28], so a simpler yet efficient version of the algorithm is derived.

The paper is organized as follows. In Section II, the signal model and the proposed structure for the receiver are presented. The joint estimation MMSE procedure for the parameters of the beamformer and the channel are presented in Section III. Section IV presents the possible options in the design of the receiver for different modulations and propagation conditions. Section V shows how the wide-band beamformer structure can be simplified under certain conditions. Finally, numerical results in Section VI illustrate the behavior of the receiver, and it is compared to other suboptimal popular structures. Section VII concludes the paper.

\section{Multichannel Single-USER ReCEIVER}

The propagation model considered below is based on the following assumptions.

- Waves impinging on the antenna array are not necessarily planar.

- The separation between sensors is one-half the carrier wavelength. The array is not necessarily linear.

- The inverse of the bandwidth of the complex envelope is much larger than the propagation time through the array.

- The user of interest and the interferers are frame synchronized, if they access a channel on a TDMA basis.

- Sampling is simultaneous in all sensors.

- Response of the array elements is constant over time.

Let the number of users be $R$, each transmitting simultaneously from a different location in space to a receiver consisting of $M$-element array of arbitrary geometry. The transmitted baseband signal for the $l$ th user is

$$
s^{(l)}(t)=\sum_{n} d^{(l)}(n) p^{(l)}(t-n T)
$$

where $d^{(l)}(n)$ are the symbols transmitted by the user $l, p^{(l)}(t)$ is the signature associated to the user and includes the effect of transmitter and receiver filters as well as the spreading signature. It reaches the array through a number of $q^{(l)}$ propagation paths, each one being characterized by a complex impulse response $c^{(l p)}(t)$ which may be, in general, frequency selective. The $q^{(l)} \times$ 1 vector of impinging signals is represented as

$$
\boldsymbol{u}^{(l)}(t)=\sum_{n} d^{(l)}(n) f^{(l)}(t-n T)
$$

where $f^{(l)}(t)$ is the vector of path responses, so that its $p$ th component is given by

$$
f^{(l p)}(t)=p^{(l)}(t) * c^{(l p)}(t)
$$

The delay associated with each user is included in $c^{(l p)}(t)$. Every path received at the array is affected by a vector describing the propagation effect across the aperture, and depends on the angle of arrival of the path and the geometry of the array. All these vectors can be arranged into an $M \times q^{(l)}$ matrix $\boldsymbol{A}^{(l)}$ (usually called the array manifold) thus resulting in a received signal

$$
\boldsymbol{x}^{(l)}(t)=\sum_{n} d^{(l)}(n) \boldsymbol{A}^{(l)} \boldsymbol{f}^{(l)}(t-n T)+\boldsymbol{v}(t)
$$

Matrix $A^{(l)}$ may also include the effects of cable and RF receiver response, I/Q imbalance, antenna elements radiation patterns, element coupling, scattering from objects near the receiver, etc. The combined effects of multipath propagation and direction of arrival give rise to the combined space-time channel

$$
\boldsymbol{g}^{(l)}(t)=\boldsymbol{A}^{(l)} \boldsymbol{f}^{(l)}(t)
$$

The received signal can then be written as a sum of terms in which we can isolate the signal of the desired user

$$
\begin{gathered}
\boldsymbol{x}(t)=\sum_{n} d^{(i)}(n) \boldsymbol{g}^{(i)}(t-n T)+\sum_{\substack{l=1 \\
l \neq i}}^{R} \sum_{n} \\
\cdot d^{(l)}(n) \boldsymbol{g}^{(l)}(t-n T)+\boldsymbol{v}(t)
\end{gathered}
$$

where the noise vector process $v(t)$ is independent of the transmitted symbols. Usually, single-user receivers consider interferent terms in the second summation as being noise, and rely either on the orthogonality of the signatures of different users or in spatial diversity, when using array receivers, for good separation [13]. Although this signal model is not strictly valid for nonlinear modulations (as is the case in GSM), in practice a reasonable linear approximation is possible [7].

For this signal model, the joint-detection optimum receiver has been developed in [12] for nondispersive channels and in [11] for dispersive channels. A suboptimal approach considers only single-user multisensor detection and takes the interference as Gaussian white noise. This receiver is often called Vector MLSE [18].

The structure proposed here is also suboptimum in the sense that interferers are considered as noise, but it exhibits a lower complexity compared to the VMLSE approach. The complete scheme is depicted in Fig. 1. As usual, a received baseband signal is passed through a filter matched to the transmission 


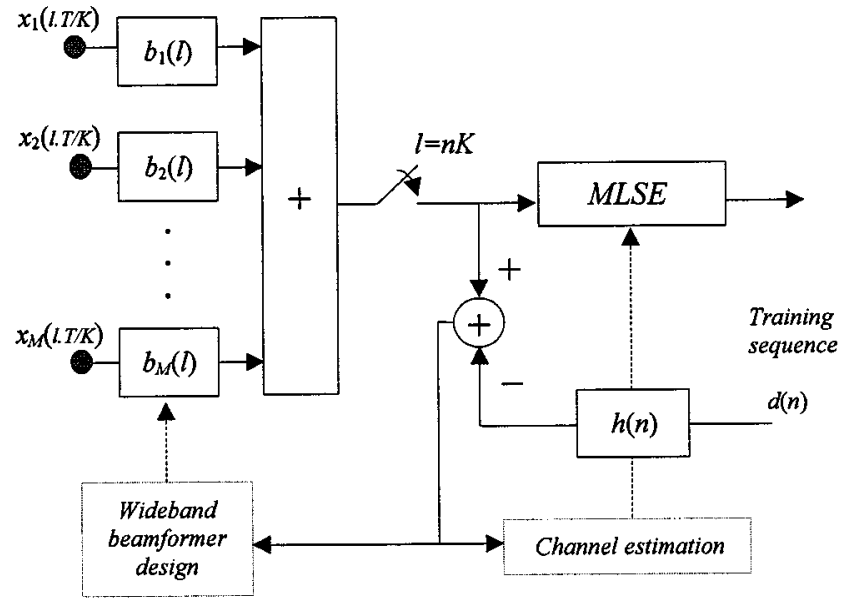

Fig. 1. The MDIR receiver, performing wideband array combining and symbol detection. A wideband spatial combiner of $K$ samples per branch is represented.

filter and sampled at the convenient sampling rate-a procedure which is not represented in Fig. 1. Those samples are combined by transversal filters $b(l)$ of length $K$, which spans at most one symbol time, followed by a sampler-by- $K$. Note that no equalization is done and the thermal noise remains temporally white at the output of the sampler. This signal is compared to the output of a filter $h(n)$ which is excited by the training sequence. The error signal is used to compute $\boldsymbol{b}$ and $\boldsymbol{h}$ using the MMSE criterion. Note that the scheme allows the removal of cochannel interferences as well as late arrival paths of the desired user by the spatial combiner that cannot be accommodated into the length of $h(n)$. At the same time, some ISI is allowed (since $h(n)$ may be several samples long) and taken into account in the MLSE processor. This feature takes advantage of time diversity, and hence robustness to fading.

\section{JOINT COMBINER AND CHANNEL RESPONSE ESTIMATION}

A training sequence of length $N$ for the desired user is needed to jointly determine the parameters of the combiner and the taps of the equivalent channel $h(n)$. Assume that the space-time channel spans its response a time interval of $(L+1) T$ seconds. The operation performed by the array combiner and the sampler during the transmission of the training sequence may be expressed as (dropping the response of the first $L-1$ symbols):

$$
\boldsymbol{X \boldsymbol { b }}=\left[\begin{array}{cccc}
\boldsymbol{x}_{1}^{T}(L) & \boldsymbol{x}_{2}^{T}(L) & \cdots & \boldsymbol{x}_{M}^{T}(L) \\
\boldsymbol{x}_{1}^{T}(L+1) & \boldsymbol{x}_{2}^{T}(L+1) & \cdots & \boldsymbol{x}_{M}^{T}(L+1) \\
\vdots & \vdots & \ddots & \vdots \\
\boldsymbol{x}_{1}^{T}(N-1) & \boldsymbol{x}_{2}^{T}(N-1) & \cdots & \boldsymbol{x}_{M}^{T}(N-1)
\end{array}\right]\left[\begin{array}{c}
\boldsymbol{b}_{1} \\
\boldsymbol{b}_{2} \\
\vdots \\
\boldsymbol{b}_{M}
\end{array}\right]
$$

where $X$ is the received signal matrix containing all users and noise (the subscripts inside $\boldsymbol{X}$ indicate the number of sensor) and $M$ is the total number of sensors. The vectors in (7) are defined as

$$
\begin{aligned}
& \boldsymbol{x}_{l}^{T}(n)=\left[\begin{array}{lll}
x_{l}(n T) & x_{l}(n T+T / K) & \cdots \\
x_{l}(n T+T(K-1) / K)
\end{array}\right] \\
& \boldsymbol{b}_{l}^{T}=\left[\begin{array}{llll}
b_{l, 0} & b_{l, 1} & \cdots & b_{l,(K-1)}
\end{array}\right] .
\end{aligned}
$$

The signal model may be conveniently written as

$$
\boldsymbol{X}=\boldsymbol{D} \boldsymbol{G}+\boldsymbol{W}
$$

with matrix $W$ containing the noise and undesired users and matrix $D$ containing the symbols of the training sequence of the desired user

$$
\boldsymbol{D}=\left[\begin{array}{cccc}
d(L) & d(L-1) & \cdots & d(0) \\
d(L+1) & d(L) & \cdots & d(1) \\
\vdots & \vdots & \ddots & \vdots \\
d(N-1) & d(N-2) & \cdots & d(N-L-1)
\end{array}\right]
$$

$G$ is the fractionally spaced space-time channel matrix for the user-of-interest

$$
\begin{aligned}
& \boldsymbol{G}=\left[\begin{array}{llllllll}
\boldsymbol{g}_{1,0} & \boldsymbol{g}_{1,1} & \cdots & \boldsymbol{g}_{1,(K-1)} & \cdots & \boldsymbol{g}_{M, 0} & \boldsymbol{g}_{M, 1} & \cdots
\end{array}\right. \\
& \left.\boldsymbol{g}_{M,(K-1)}\right] \\
& G \in \mathbb{C}^{(L+1) \times M K} \text {. }
\end{aligned}
$$

Note that $\boldsymbol{g}_{j, r}$ contains the channel impulse response between the user-of-interest and sensor $j$ at the sampling phase $n T+$ $r T / K$. It is implicitly assumed that the interferers are all framesynchronized and their training sequences are sufficiently uncorrelated to allow separation on a time-reference basis. In the sequel, we make the approximation that the noise-plus-interference matrix $W$ is spatially and temporally white.

The joint design of the spatial combiner $\boldsymbol{b}$ and the impulse response $\boldsymbol{h}$ for the sequence detector is based on the minimization of the mean square error $\eta$

$$
\eta=\|\boldsymbol{X} \boldsymbol{b}-\boldsymbol{D} \boldsymbol{h}\|_{2}^{2} .
$$

Note that this optimization is aimed at keeping the multipath content of the signal so that it can be used later by the MLSE processor. A proper constraint has to be imposed in order to avoid the trivial solution. Several options are available [3]. In [5], the term $\boldsymbol{h}^{H} \boldsymbol{h}$ was fixed. Experimentally, the one proving more efficient is based on the control of the desired signal energy at the output of the spatial combiner, which can be formulated as

$$
E=\boldsymbol{b}^{H} \boldsymbol{G}^{H} \boldsymbol{D}^{H} \boldsymbol{D} \boldsymbol{G} \boldsymbol{b} .
$$

We force this value to a nonzero constant $\alpha$. The application of a regular minimization procedure to the Lagrangian function

$$
J=\eta-\lambda\left(\boldsymbol{b}^{H} G^{H} \boldsymbol{D}^{H} \boldsymbol{D} G \boldsymbol{b}-\alpha\right)
$$

leads to the following two equations:

$$
\begin{gathered}
h=\left(\boldsymbol{D}^{H} \boldsymbol{D}\right)^{-1} \boldsymbol{D}^{H} \boldsymbol{X} \boldsymbol{b} \\
\boldsymbol{X}^{H}\left(\boldsymbol{I}-\boldsymbol{D}\left(\boldsymbol{D}^{H} \boldsymbol{D}\right)^{-1} \boldsymbol{D}^{H}\right) \boldsymbol{X} \boldsymbol{b}=\lambda G^{H} \boldsymbol{D}^{H} \boldsymbol{D} \boldsymbol{G} \boldsymbol{b} .
\end{gathered}
$$

If the training sequence and the noise-plus-interference are uncorrelated processes, then we can consider that, for $N-L$ sufficiently large,

$$
D^{H} \boldsymbol{X} \cong \boldsymbol{D}^{H} \boldsymbol{D} \boldsymbol{G}
$$


which allows to rewrite (15a) and (15b) as follows:

$$
\begin{aligned}
\boldsymbol{h} & =\boldsymbol{G} \boldsymbol{b} \\
\boldsymbol{R}_{w} \boldsymbol{b} & =\lambda \boldsymbol{G}^{H} \boldsymbol{D}^{H} \boldsymbol{D} \boldsymbol{G} \boldsymbol{b}
\end{aligned}
$$

where the noise-plus-interference matrix $\boldsymbol{R}_{w}$ is defined as

$$
\boldsymbol{R}_{w}=\boldsymbol{X}^{H} \boldsymbol{X}-\boldsymbol{G}^{H} \boldsymbol{D}^{H} \boldsymbol{D} \boldsymbol{G} .
$$

Equation (17a) is an expected result: the impulse response seen by the MLSE block is the space-time channel filtered by the array combiner. The SINR at the output of the sampler is given by

$$
\operatorname{SINR}=\frac{\boldsymbol{b}^{H} \boldsymbol{G}^{H} \boldsymbol{D}^{H} \boldsymbol{D} \boldsymbol{G} \boldsymbol{b}}{\eta_{\mathrm{opt}}}=\frac{\boldsymbol{b}^{H} G^{H} \boldsymbol{D}^{H} \boldsymbol{D} G \boldsymbol{b}}{\boldsymbol{b}^{H} \boldsymbol{R}_{w} \boldsymbol{b}}=\frac{1}{\lambda}
$$

Therefore, the coefficients of the linear combiner $\boldsymbol{b}$ are given by the generalized eigenvector of (17b) associated with the minimum generalized eigenvalue. From (19), it is concluded that the eigenvalues are positive. On the other hand, (17a) reveals that the impulse response of the channel that is to be used in the MLSE block is the matched response of the linear combiner plus the sampler to the physical channel (hence giving a name to the receiver).

Care has to be taken to guarantee that matrix $\boldsymbol{R}_{w}$ is full rank, otherwise the computation of the generalized EVD is not a wellconditioned problem. Inspecting (18) when number of rows in matrix $\boldsymbol{X}(N-L)$ is smaller than the number of columns $M K$ (that is, the number of elements of $\boldsymbol{b}$ ), the product $\boldsymbol{X}^{H} \boldsymbol{X}$ is rank deficient and so is $\boldsymbol{G}^{H} \boldsymbol{D}^{H} \boldsymbol{D} \boldsymbol{G}$. Good performance has been observed when diagonal loading is used on matrix $\boldsymbol{R}_{w}$ :

$$
\boldsymbol{R}_{w} \leftarrow \boldsymbol{R}_{w}+\sigma^{2} \boldsymbol{I}
$$

by giving a value to $\sigma^{2}$ just below the ground noise level. The degree of load is not critical, but note that a large value would imply that the most important undesired signal is white noise and would result in a distorted beamformer pattern.

Equation (16) shows the procedure used to estimate the space-time signal channel $\boldsymbol{G}$. Of course, if the symbols of the training sequence are uncorrelated, then $\boldsymbol{D}^{H} \boldsymbol{D} \cong(N-L) \boldsymbol{I}$, and previous equations can be further simplified.

The ability to null interferers, as well as late arrivals of the signal of interest, is strongly dependent on two facts.

1) Consider the number of elements of the array and the spatial dispersion of the signals and interferers. It is widely known [14] that an $M$-element array is able to cancel up to $M-1$ interferer paths. The direct consequence of this fact is that a high number of interfering signals in highly spatially dispersive channels cannot be fully cancelled and limits the performance of the proposed scheme since time correlated noise (interference) $w(n)$ is induced at the output of the sampler. This noise might be dealt with at the MLSE block by using its covariance matrix in the computation of the metrics in the Viterbi lattice.

2) The use of a fractionally spaced beamformer allows further interference rejection [27] provided that the incoming signal has some excess bandwidth.

\section{Design Options AND TRAdeoffS}

The MDIR receiver presented above is flexible enough to allow different configurations depending on the air interface or the propagation conditions. The following are some design options.

- Nonspread modulation, whereby the channel usually spans several symbols. The filters of the broad-band spatial combiner span one symbol time and $K$ might be 1 or 2 samples. In spite of the lack of excess bandwidth of GMSK, the improvement of taking 2 samples per symbol may be significant [27].

- Spread modulation-in this case, there are two options.

1) The filter length at each sensor spans one chip and $K=1,2 \cdots$, depending on the signal bandwidth. In this case, the impulse response $\boldsymbol{h}$ is estimated at chip time. If the impulse response is short with respect to the symbol time, a one-shot RAKE combiner plus a detector is a good approximation to the MLSE processor. In the case of wide-band CDMA (the impulse response spans more than a symbol), a complete MLSE processor with a Viterbi algorithm is needed.

2) The filter length at each sensor spans a multiple of the spreading factor, $K=\mathrm{SF}, 2 \mathrm{SF}, \cdots$, depending on the signal bandwidth. The array combiner becomes a decorrelating receiver, but note that no equalization is done since the filters length is one symbol time and the sampler follows the spatial combiner. Therefore, the array has further potential of users separation. If the channel impulse response is short with respect to the spreading factor, a simple level detector can efficiently substitute the MLSE processor. Otherwise, a symbol level MLSE block with a Viterbi algorithm is needed.

\section{COMPLEXITY Reduction IN THE Wide-BAND BEAMFORMING}

Wide-band spatial combining allows additional signal cancellation because it performs a different narrow-band beamformer per delay. However, as the signal scenario presents low angular dispersion, it is reasonable to assume that all multipath rays with its associated time delays come from a narrow solid angle, and therefore each user can be considered a point source. In this case, there is no need to build a different narrow-band combiner per delay and all filters $\boldsymbol{b}_{l}$ present at the branches of the array are the same. The scheme for the receiver in this case is given in Fig. 2.

Since this is a particular case of the more general problem presented in Section II, it is to be expected that the solution found in Section III can be accommodated to this model. Assume again that samples at a rate $K / T$ are fed to the linear combiner. Then let us write the output of the linear combiner plus sampler as

$$
y(n)=\boldsymbol{x}^{T}(n) \boldsymbol{b}=\left[\begin{array}{llll}
\boldsymbol{x}_{1}^{T}(n) & \boldsymbol{x}_{2}^{T}(n) & \cdots & \boldsymbol{x}_{M}^{T}(n)
\end{array}\right]\left[\begin{array}{c}
\boldsymbol{b}_{1} \\
\boldsymbol{b}_{2} \\
\vdots \\
\boldsymbol{b}_{M}
\end{array}\right]
$$




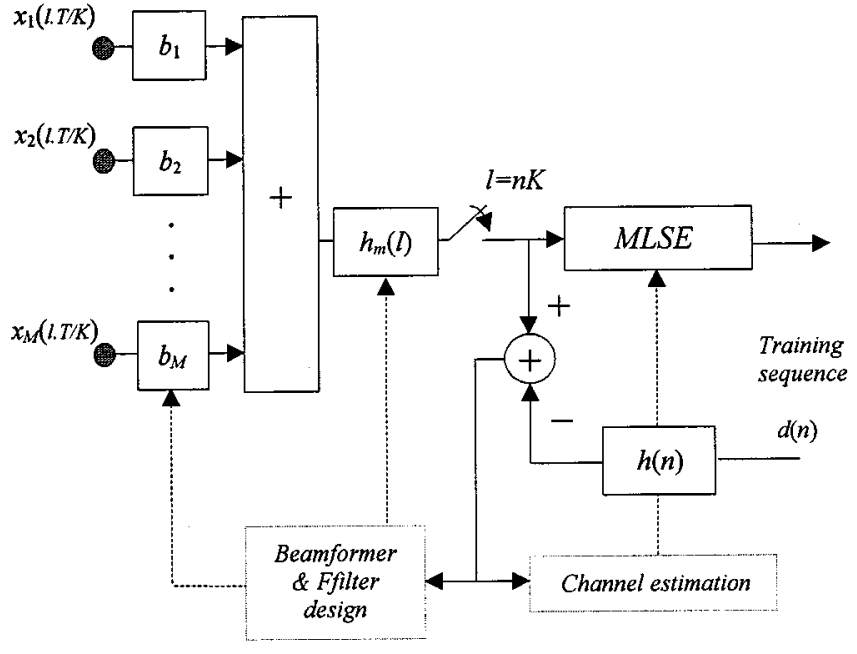

Fig. 2. The proposed spatial combiner, including a single linear filter, suitable for low dispersive channels.

$$
=\operatorname{trace}\left(\boldsymbol{X}(n)^{T} \boldsymbol{B}\right)
$$

where

$$
\begin{aligned}
& X(n)=\operatorname{unved}(\boldsymbol{x}(n)) \quad \in \mathbb{C}^{K \times M} \\
& B=\text { unved }(\boldsymbol{b}) \quad \in \mathbb{C}^{K \times M} \text {. }
\end{aligned}
$$

Now, the beamformer plus matched filter is obtained as rank-one approximation of the matrix $B$ :

$$
\boldsymbol{B}=\sum_{i=1}^{\min (K, M)} \sigma_{i} \boldsymbol{u}_{i} \boldsymbol{v}_{i}^{H} \cong \sigma_{\max } \boldsymbol{u}_{\max } \boldsymbol{v}_{\max }^{H} .
$$

The left and right eigenvectors associated with the maximum eigenvalue provide the coefficients of the linear filter $\boldsymbol{h}_{m}$ and the conjugate beamformer coefficients $\boldsymbol{b}$, respectively. The goodness of this approximation may be decided from the quotient between the maximum eigenvalue and the sum of singular values of $B$. The truncation in (24) can be made on any order (not just keeping one term), therefore allowing simplification of the receiver according to the characteristics of the scenario.

\section{Performance Evaluation}

Since the MDIR receiver is a suboptimal single-user receiver, we will compare its performance to two practical single-user approaches usually proposed. Both consider interferers as Gaussian noise.

1) The multisensor vector MLSE (VMLSE) [18] is a popular, reasonably priced receiver which models the noise plus interference as spatially and emporally white.

2) The multisensor weighted vector MLSE (WVMLSE) receivers [25], [26], are based on a modification of the vector MLSE metrics by using the inverse of the space correlation matrix of the interference. This matrix can be readily estimated as

$$
\hat{\boldsymbol{R}}_{w}=(\boldsymbol{X}-\boldsymbol{D} \boldsymbol{G})^{H}(\boldsymbol{X}-\boldsymbol{D} \boldsymbol{G}) .
$$

This receiver is a fair competitor of MDIR since it performs some kind of spatial cancelling, but at a higher complexity. No time modeling of the interference has been attempted since it would imply a larger number of states in the Viterbi algorithm [24].

\section{A. Propagation Channel Model}

In order to evaluate the receiver in a realistic mobile scenario, we have carried out simulations based on a Gaussian stationary uncorrelated hypothesis for the channel, assuming independence between angular and Doppler spread, as it has been experienced from measurements taken in downtown Stockholm in the $1.8 \mathrm{GHz}$ band [10]. There, it is empirically shown that azimuth spectrum follows a Laplacian law, along with Gaussian distribution for the directions of arrival $(\phi)$ around the mean angular position of the user. The angular spread (that is, the standard deviation of the Gaussian, $\sigma_{\phi}$ ) is taken to be $8^{\circ}$. The number of rays impinging the array is fitted as a Poisson random variable of mean 25. An exponential law is found in [10] for the power delay spread. The delay associated with each impinging ray $(\tau)$ is taken as an exponential random variable of mean $1 \mu \mathrm{s}$ $\left(\sigma_{\tau}\right)$. The amplitude associated with each propagation path $(\alpha)$ is a complex Gaussian random variable whose power decreases as the time delay and the angular direction of arrival with respect to the mobile position increase, according to the expression

$$
E\left\{|\alpha|^{2} / \tau, \phi\right\}=\exp \left(-\frac{\tau}{6.88 \sigma_{\tau}}+\frac{\phi^{2}}{3.81 \sigma_{\phi}^{2}}-\sqrt{2} \frac{|\phi|}{\sigma_{\phi}}\right) .
$$

A classic Clarke's bath-shaped Doppler spectrum is obtained by assuming multiple reflections close around the mobile. The array works in a sectored area of $120^{\circ}$, with linearly and uniformly spaced at $d / \lambda=0,5$. All plots shown in the simulations below are representations of the performance of the link level which can be used later, through convenient mapping, to obtain FER (frame erasure ratio) when considering channel coding or other system level features (like power control, frequency hopping, or discontinuous transmission) [20].

\section{B. Simulation 1: Nonspread Modulation}

The air interface is TDMA-based, and training sequences are formed, as in the GSM standard [7], by 26 symbols located in the middle of 116 traffic symbols frame. BPSK modulation with rolloff factor 0.2 has been used. Co-channel users in other cells are the source of interference. It is assumed that they are framesynchronous, with relative delays of up to two symbols. This implies base-station synchronicity. The length of the estimated channel is $L+1=4$.

First, the three receivers (MDIR, VMLSE, and WVMLSE) have been compared with respect to the number of interferent signals, versus the frame instantaneous CIR, using 4 and 8 sensors; 3 and 6 interferers have been tested, all of equal mean power, which can be considered a pessimistic signal scenario when cells are sectored in $120^{\circ}$. The mean $E_{b} / N_{\circ}$ is $25 \mathrm{~dB}$ and the mobile speed is a vector of modulus $50 \mathrm{~km} / \mathrm{h}$ and random direction. Training sequences are taken from the GSM standard [9]. Note that they are not completely orthogonal. The number of samples per symbol is one, which is the length of the filters at each branch of the array, and the right sampling time has 

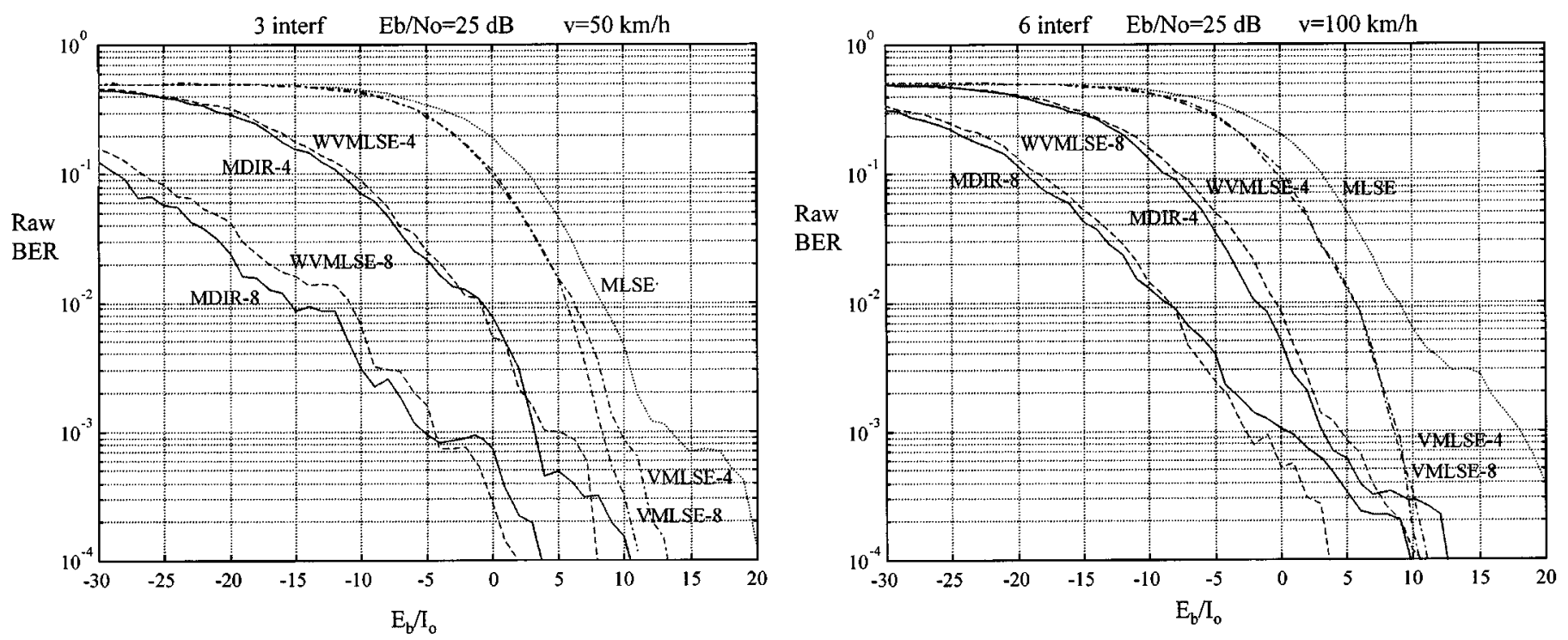

Fig. 4. Probability of error of all receivers with increased mobile speed.

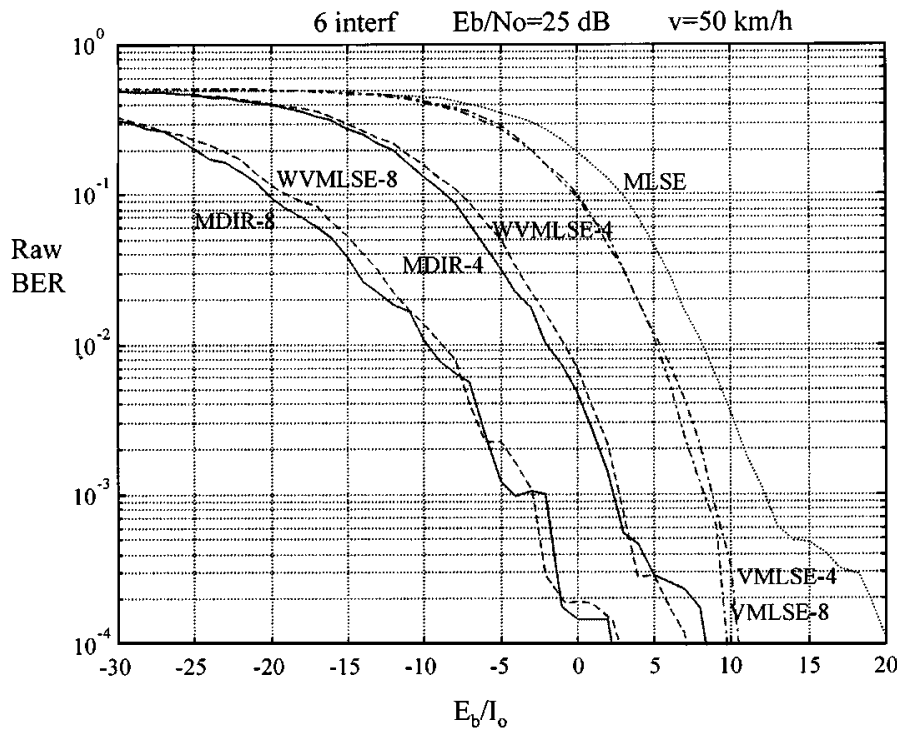

In the third simulation, the mean $E_{b} / N_{o}$ level has been changed for each receiver in values from 5 to $35 \mathrm{~dB}$. As expected, the VMLSE shows the best performance when the interference levels are very low. WVMLSE should exhibit similar BER values in this condition, but the estimation error in matrix $\hat{\boldsymbol{R}}_{w}$ is significant, due to the short length of the training sequence. On the contrary, when the $E_{b} / N_{o}$ is high, VMLSE exhibits some limiting performance with respect to interference cancellation, which is not found for MDIR and WVMLSE.

\section{Simulation 2: Spread Modulation}

In order to test the potentials of the receiver when using excess bandwidth signals, the same signals are spread with equal length spreading codes for each user. Chip time is $0.9 \mu$ s. Except for the bandwidth, these conditions are similar to the TDD air interface proposed for the third-generation mobile communica-

Fig. 3. Probability of error of the MDIR (solid), VMLSE (dashed-dotted), and WVMLSE (dashed) receivers versus instantaneous CIR for three (upper) and six (lower) interferent signals. The performance of the single-sensor MLSE is also displayed (dotted). Four and eigth sensors have been tested.

been computed out from the channel estimated at 4 samples per symbol.

Plots showing the raw BER are displayed in Fig. 3, where the estimated BER does not include the detected symbols of the training sequence. The results are compared to the single-sensor MLSE detector versus the instantaneous CIR in the slot. According to the figures, the CIR rejection of the MDIR is similar to the WVMLSE and its gain can be quantified in $10 \mathrm{~dB}$ at $0,1 \%$ BER when using 4 antennas with respect to VMLSE. Note that WVMLSE requires a higher computational cost.

In a second simulation, we have studied the dependence on the mobile speed. Fig. 4 is equivalent to the lower graph in Fig. 3 except for the mobile speed, which is set to $100 \mathrm{~km} / \mathrm{h}$. All receivers tend to exhibit some residual BER at high CIR due to the inaccuracy in the channel estimate, except for the VMLSE. MDIR seems to be the most affected. tions system [8]. The number of taps at each sensor of the array is a multiple of the spreading factor: one or two samples per chip are taken $(K=4$ or $K=8)$. After the spatial combiner, a symbol-time sampler precedes the MLSE block. The codes chosen are orthogonal Walsh codes, and they have been taken randomly for each user in each Monte Carlo run. The channel model is the same as in previous simulations. Pulse shaping is raised cosine with rolloff factor of 0.2 . Three users of spreading factor 4 have been used, two of them being considered as asynchronous interference users. The floor noise is $20 \mathrm{~dB}$ below the mean power of the desired user and may accommodate high spreading factor users in a multirate system. The broad-band spatial combiner allows the design of a interference cancellation filter $\boldsymbol{b}$ in each array branch, which further reduces the interference level at the input of the MLSE block. The BER is displayed in Fig. 6 along with the performance of the receiver with chip-time sampler after the spatial combiner, and using one or two samples per chip ( $K=1$ and $K=2$ ). From the figure, it is apparent that the broad-band combiner with symbol-time sampling allows a performance with one single sensor that is comparable to the performance of a four sensor array using chip-time 


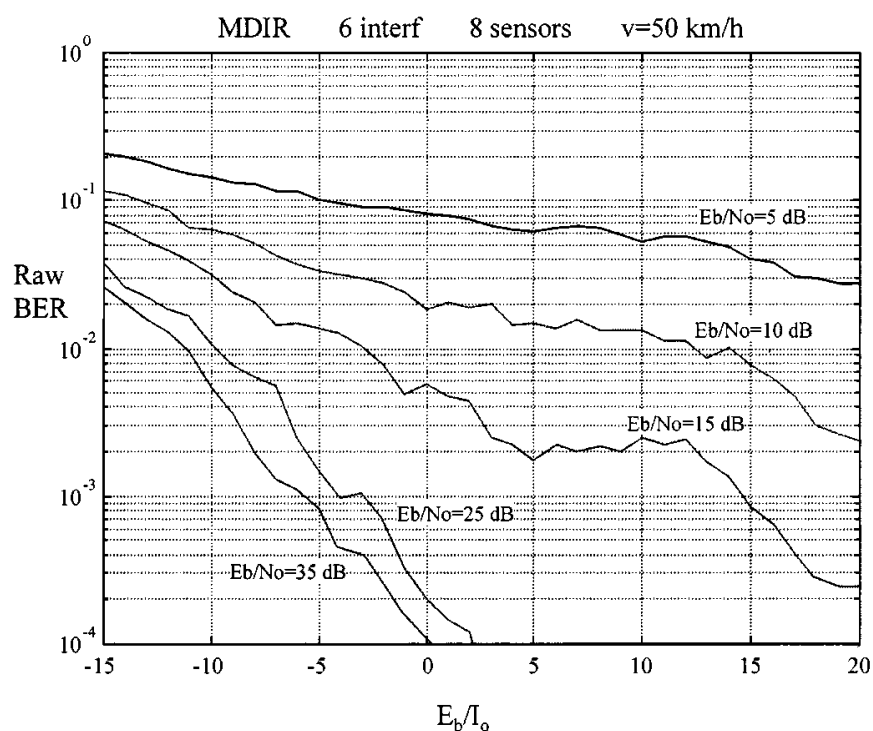

(a)

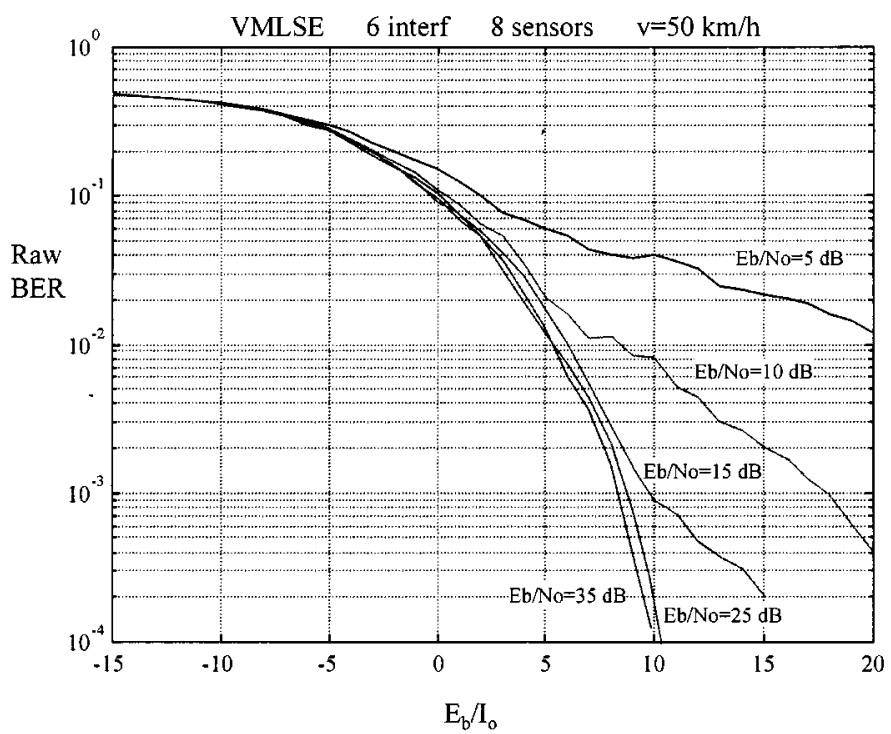

(b)

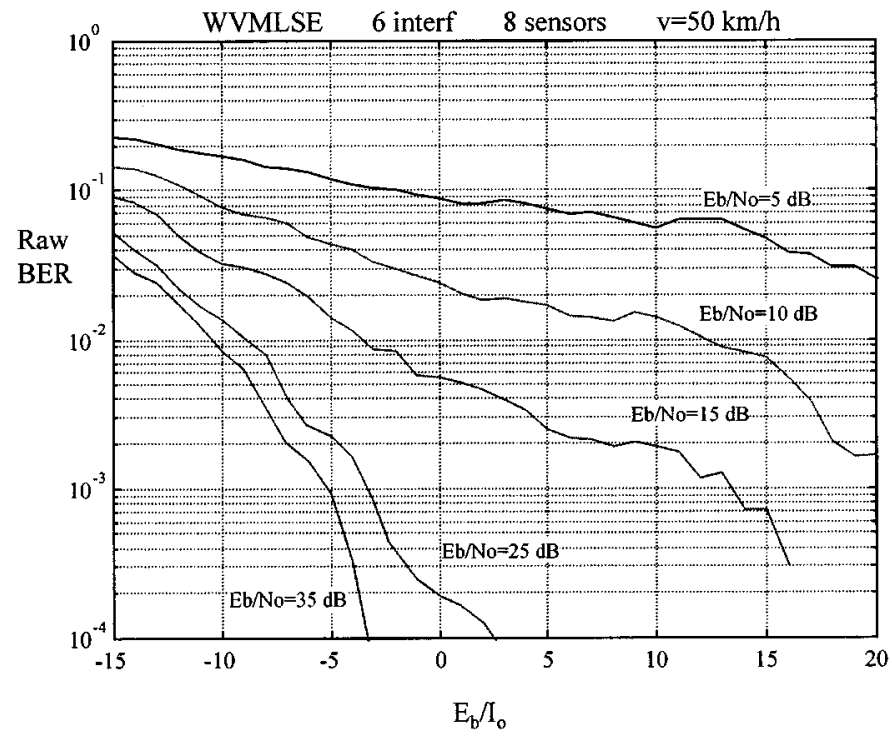

(c)

Fig. 5. Probability of error of the (a) MDIR, (b) VMLSE, and (c) WVMLSE receivers versus instantaneous CIR for six interferers, a mobile speed of $50 \mathrm{~km} / \mathrm{h}$ and different mean $E_{b} / N_{o}$ values. Eight sensors have been used in all cases.

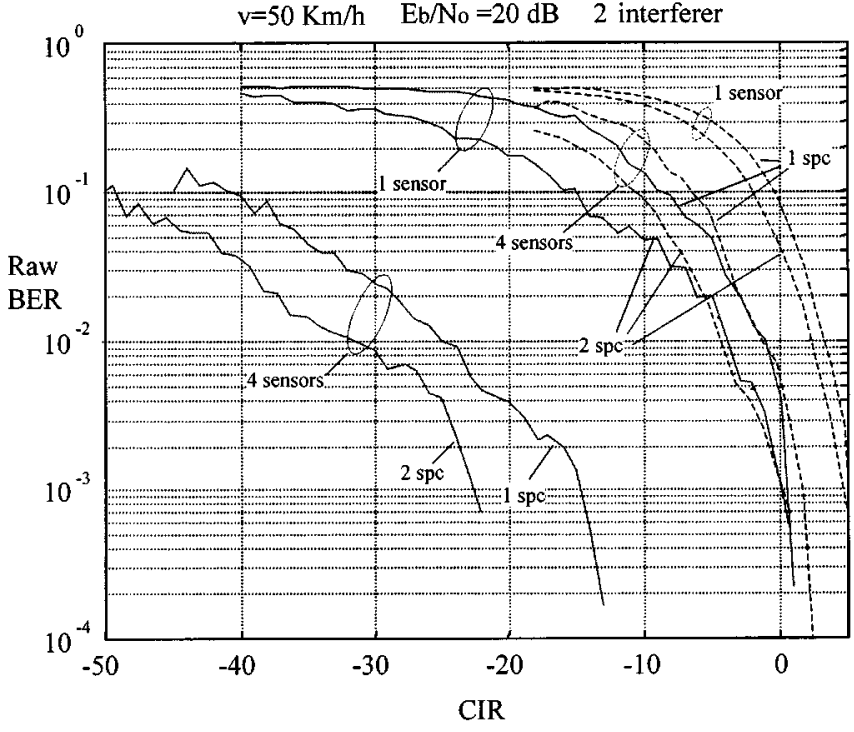

Fig. 6. Probability of error of the MDIR (solid) and VMLSE (dashed) receivers versus instantaneous CIR in DS/SS channel access with spreading factor of 4. Two interferer users are considered. One and four sensors have been used, each featuring a linear filter spanning one symbol time and 1 or 2 samples per chip ( $1 \mathrm{spc}$ or $2 \mathrm{spc}$ ), so the number of coefficients is $K=4$ or 8 .

signal as input to the MLSE. No significant gains are observed when four samples per symbol are used.

\section{CONCLUSION}

A simple and efficient receiver combining temporal and spatial diversity has been presented. Good performance of the receiver relies on the fact that enough sensors and taps of the matched impulse response are available to null interferences and late arrivals of the desired user, prior to an MLSE which acts as a temporal processor. Simulations have been carried out with accurate models of mobile channels showing that the proposed receiver largely outperforms the VMLSE receiver in a wide range of CIR at a lower complexity, and has a similar performance to the WVMLSE but a lower computational requirements.

By using eight sensors in single interference scenario, the proposed receiver extends the robustness of the single sensor significantly. Simulations have shown that oversampling may give additional gains. When used in a digital cellular mobile systems, this figure translates into a superior robustness to interference level and hence allows for a tighter frequency planning (cluster size reduction), with the subsequent increase in systems capacity.

\section{REFERENCES}

[1] P. D. Shaft and E. C. Kather, "Experimental measurement of Viterbi decoding in burst channels," IEEE Trans. Commun., vol. COM-27, pp. 1360-1366, Aug. 1978.

[2] D. R. Falconer and F. R. Magee, "Evaluation of decision feedback equalization and Viterbi algorithm detector for voice band data transmission," IEEE Trans. Commun., vol. COM-24, pp. 1130-1139, Oct. 1976.

[3] F. R. Magee, "A comparison of compromise Viterbi algorithm and standard equalization techniques over band limited channels," IEEE Trans. Commun., vol. COM-23, pp. 361-367, Mar. 1975.

[4] M. A. Lagunas, A. I. Perez, and J. Vidal, "Joint beamforming and Viterbi equalizers in wireless communications," in Proc. 31st Asilomar Conf. Signals, Syst. Computers, Nov. 1997. 
[5] F. Pipon, P. Chevalier, P. Vila, and J. J. Monott, "Joint spatial and temporal equalization for channels with ISI and ICI: Theoretical and experimental results for a base-station," in Proc. IEEE Workshop Signal Advances Wireless Commun. SPAWC'97, Paris, Apr. 1997, pp. 309-312.

[6] F. Falconer and R. Magee, "Adaptive channel memory truncation for maximum likelihood sequence estimation," Bell Syst. Tech. J., vol. 9, pp. 1541-1562, Nov. 1973.

[7] R. Steele, Mobile Radio Communications. New York: Pentech, 1992.

[8] ETSI, "Evaluation report for ETSI UMTS terrestrial radio access (UTRA) ITU-R RTT candidate," Tech. Rep. ETSI-SMG2, 1998.

[9] European Telecommunications Standard, ,p. 12, Aug. 1975.

[10] K. Pedersen, P. Mogensen, and B. Fleury, "A stochastic model of the temporal and azimuthal dispersion seen at the base station in outdoor propagation environments," IEEE Trans. Veh. Technol., vol. 49, no. 2, Mar. 2000.

[11] M. Stojanovic and Z. Zvonar, "Multichannel processing of broad-band multiuser communication signals in shallow water acoustic channels," IEEE J. Oceanic Eng., vol. 21, Apr. 1996.

[12] S. Y. Miller and S. C. Schwartz, "Integrated spatial-temporal detectors for aynchronous Gaussian multiple-access channels," IEEE Trans. Commun., vol. 43, Feb./Mar./Apr. 1995.

[13] H. V. Poor and S. Verdu, "Single-user detectors for multiuser channels," IEEE Trans. Commun., vol. 36, pp. 50-60, Jan. 1988.

[14] R. A. Monzingo and T. W. Miller, Introduction to Adaptive Arrays. New York: Wiley, 1980.

[15] J. Proakis, Digital Communications. New York: McGraw-Hill, 1995.

[16] J. W. Liang and A. Paulraj, "Two stage CCI/ISI reduction with space-time processing in TDMA cellular networks," in Proc. 30th Annu. Asilomar Conf. Signals, Syst. and Computers, Pacific Grove, CA, Nov. 1996.

[17] A. J. Paulraj and C. B. Papadias, "Space-time processing for wireless communications," IEEE Signal Processing Mag., pp. 49-83, Nov. 1997.

[18] W. Van Etten, "Maximum likelihood receiver for multiple channel transmission systems," IEEE Trans. Commun., pp. 276-283, Feb. 1976

[19] B. Suard, G. Xu, H. Liu, and T. Kailath, "Uplink channel capacity of space-division multiple-access schemes," IEEE Trans. Info. Theory, vol. 44, pp. 1468-1476, July 1998.

[20] S. Hämäläinen, P. Slanina, M. Hartman, A. Lappeteläinen, H. Holma, and O. Salonaho, "A novel interface between link and system level simulations," in Proc. ACTS Mobile Telecommun. Summit, Aalborg, Denmark, Oct. 1997, pp. 599-604.

[21] P. Balaban and J. Salz, "Optimum diversity combining and equalization in digital data transmission with applications to cellular mobile radio-Part I: Theoretical considerations," IEEE Trans. Commun., vol. 40, pp. 885-907, May 1992.

[22] TSUNAMI II (ACTS 020) Rep., "Algorithms and antenna array recommendations (Part 1)," AC020/AUC/A1.2/DR/P/005/b1, May 1997.

[23] G. E. Bottomley and S. Chennakeshu, "Unification of MLSE receivers and extension to time-varying channels," IEEE Trans. Commun., vol. 46, pp. 464-472, Apr. 1998.

[24] M. Rupf, F. Tarköy, and J. L. Massey, "User-separating demodulation for code-division multiple access systems," IEEE J. Select. Areas Commun., pp. 786-795, June 1994.

[25] G. Bottomley and K. Jamal, "Adaptive arrays and MLSE equalization," in Proc. 45th IEEE Veh. Technol. Conf., Chicago, 1995, pp. 50-54.

[26] M. Escartin and P. A. Ranta, "Interference rejection with a small antenna array at the mobile scattering environment," in Proc. IEEE Signal Processing Workshop Signal Processing Advances Wireless Commun., Paris, Apr. 1997, pp. 165-168.

[27] J. C Liberti and T. S. Rappaport, Smart Antennas for Wireless Communications: IS-95 and Third Generation CDMA Applications. Englewood Cliffs, NJ: Prentice-Hall, 1999

[28] M. Stojanovic, J. A. Catipovic, and J. G. Proakis, "Reduced-complexity spatial and temporal processing of underwater acoustic communication signals," J. Acoust. Soc. Amer., pt. 1, vol. 98, no. 2, pp. 961-972, Aug. 1995
Miguel A. Lagunas (S'73-M'78-SM'89-F'97) was born in Madrid, Spain, in 1951. He received the Telecommunications Engineer degree in 1973 from UPM, Madrid, and the Ph.D. degree in Telecommunications from UPB, Barcelona.

From 1971-1973, he was a Research Assistant at the Semiconductor Lab ETSIT, Madrid; from 1973-1979, a Teacher Assistant in Network Synthesis and Semiconductor Electronics; from 1979-1982, an Associate Professor of digital signal processing. Since 1983, he has been a Full Professor, teaching courses in signal processing, array processing and digital communications. He was Project Leader of high-speed SCMA (1987-1989) and ATM (1994-1995) cable network. He is also Co-director of the first projects for the European Spatial Agency and the European Union, providing engineering demonstration models on smart antennas for satellite communications using DS and FH systems (1986) and mobile communications GSM (Tsunami, 1994). His research activity is devoted to spectral estimation, adaptive systems, and array processing. His technical activities are in advanced front-ends for digital communications combining spatial with frequency-time and coding diversity.

Dr. Lagunas was Vice-President for Research of (UPC) from 1986-1989 and Vice-Secretary General for Research, Cicyt, Spain from 1995-1996. He is a member-at-large of Eurasip. He is an elected member of the Academy of Engineers of Spain. He was a Fullbright scholar at the University of Boulder, CO.

Josep Vidal (S'88-M'91) was born in Barcelona, Spain, in 1965. He received the telecommunication engineering and the $\mathrm{Ph} . \mathrm{D}$. degrees from the Polytechnic University of Catalonia (UPC), Barcelona, in 1989 and 1993, respectively. In 1988, he was with Cognivision Research, S.L. developing hardware for image analysis.

From 1989 to 1990, he was with the LSI at the Ecole Polytechnique de Lausanne as a Research Assistant, working on digital signal processing for biomedical applications. In 1991, he was recipient of a Ministry of Education grant to complete the Ph.D. thesis and joined the Signal Theory and Communications Department at UPC. In 1993, he became a Lecturer. Since 1996, he has been an Associate Professor at UPC where he teaches graduate and undergraduate courses in statistical signal processing, digital signal processors, mathematical methods for communications and non-linear signal processing. His current research interests are in statistical signal processing with application to mobile communication systems and digital watermarking, as well as DSP implementation aspects. He has published over 40 papers in these areas in international journals and conferences.

Dr. Vidal was co-organizer of the IEEE Signal Processing/ATHOS Workshop on Higher-Order Statistics in Begur, Spain, in June 1995.

Ana I. Pérez-Neira (S'92-M'95) was born in Zaragoza, Spain, in 1967. She graduated in telecommunication engineering in 1991, and received the Ph.D. degree in 1995 from the Universitat Politècnica de Catalunya (UPC), Barcelona, Spain.

In 1991, she joined the Department of Signal Theory and Communication of the UPC, where she carried on research activities in the field of higher order statistics and statistical array processing. In 1992, she became Lecturer. Since 1996, she has been an Associate Professor at UPC, where she teaches and coordinates graduate and undergraduate courses in statistical signal processing, analog and digital communications, mathematical methods for communications, and nonlinear signal processing. Her current research interests are in statistical signal processing and fuzzy processing, with applications to mobile/satellite communication systems. 